Research Article

\section{A Systematic review for sudden cardiac death in hypertrophic cardiomyopathy patients with Myocardial Fibrosis: A CMR LGE Study}

\author{
Sahadev T Reddy ${ }^{1}$, Antonio T Paladino ${ }^{2}$, Nackle J Silva ${ }^{3}$, Mark \\ Doyle ${ }^{1}$, Diane A Vido ${ }^{1}$ and Robert WW Biederman ${ }^{1 *}$
}

${ }^{1}$ Carnegie Mellon University, Allegheny Health Network, Allegheny General Hospital, Pittsburgh, PA 15212, USA

2Department of Cardiology, Dante Pazzanese Institute of Cardiology, Adib Jatene Foundation, dantepazzanese, Brazil

${ }^{3}$ Brazilian Institute of Science and Technology Information, SAUS Quadra 05 Lot 06 Block H, Asa Sul, Brazil

\section{Abstract}

Background: Hypertrophic cardiomyopathy $(\mathrm{HCM})$ patients have a predisposition for malignant VT/VF and consequently, sudden cardiac death (SCD). In single center studies, late gadolinium enhancement (LGE) defined fibrosis has been linked to VT/VF. However, despite innumerable investigations, SCD has not been definitely attributable to LGE. Explanations for these are believed to be related to insufficient statistical power.

Methods: We performed an electronic search of MEDLINE, PubMed: and CMR abstracts for original data published or presented between Jan 2001 to Mar 2011. Key search terms: HCM, LV fibrosis, SCD and LGE. Studies were screened for eligibility based on inclusion criteria: referral for CMR exam with LGE for HCM; and follow-up for incidence of VT/VF and SCD. Categorical variables were evaluated between patient groups via Chi-square test.

Results: A total of 64 studies were initially identified. Of these, 4 (6.3\%) were identified and included ( $n=1063$ patients). Three prospective and one retrospective study were included. LGE was detected in $59.6 \%$ of patients. As expected, the presence of myocardial fibrosis was associated with VT/VF $\left(x^{2}=6.5, p<0.05\right.$; OR 9.0, (95\% Cl 1.2 to 68.7$)$. Moreover, myocardial fibrosis strongly predicted $\operatorname{SCD}\left(x^{2}=6.6, p<0.05\right.$; OR $3.3(95 \% \mathrm{Cl} 1.2$ to 9.7$)$.

Conclusion: Despite single center CMR studies, LGE has consistently predicted VT/VF while prediction of SCD has remained paradoxically unlinked. Although the lack of studies meeting our criteria limited our ability to perform a comprehensive meta-analysis, we have been able to demonstrate for the first time that LGE-defined fibrosis is a predictor of SCD in patients with HCM.

\section{More Information}

*Address for Correspondence: Robert WW Biederman, MD, FACC, FAHA, FSGC, FASA, Professor of Medicine, Director, Cardiovascular Magnetic Resonance Imaging, Temple University School of Medicine, Adjunct Professor of Bioengineering, Carnegie Mellon University, Allegheny Health Network, Allegheny General Hospital, 320 E. North Avenue, Pittsburgh, PA 15212, USA, Tel: 412-359-8705; Fax: 412-359 6358; Email: RBIEDERM@wpahs.org

Submitted: 01 October 2019 Approved: 01 November 2019 Published: 04 November 2019

How to cite this article: Reddy ST, Paladino AT, Silva NJ, Doyle M, Vido DA, et al. A Systematic review for sudden cardiac death in hypertrophic cardiomyopathy patients with Myocardial Fibrosis: A CMR LGE Study. J Cardiol Cardiovasc Med. 2019; 4: 187-191.

DOI: dx.doi.org/10.29328/journal.jccm. 1001064

Copyright: (c) 2019 Reddy ST, et al. This is an open access article distributed under the Creative Commons Attribution License, which permits unrestricted use, distribution, and reproduction in any medium, provided the original work is properly cited.

Keywords: Hypertrophic cardiomyopathy; Myocardial fibrosis; Late gadolinium enhancement; Cardiac magnetic resonance imaging; Sudden cardiac death

Abbreviations: HCM: Hypertrophic Cardiomyopathy; SCD: Sudden Cardiac Death; VT/VF: Ventricular Tachycardia/Ventricular Fibrillation; LGE: Late Gadolinium Enhancement; AICD: Automatic Implantable Cardiac Defibrillator; CMR: Cardiac Magnetic Resonance Imaging

Check for updates

\section{Introduction}

Hypertrophic cardiomyopathy (HCM) is the most common cardiac genetic disorder in clinical practice that affects approximately $0.2 \%$ of general population [1]. By definition, HCM is characterized by the presence of myocardial hypertrophy in the absence of obvious cause for hypertrophy including aortic stenosis, hypertension, and other genetic or acquired infiltrative disorders. HCM is a heterogeneous disease caused by mutations in genes that encode cardiac sarcomere proteins and is transmitted through autosomal dominant trait $[2,3]$. These mutations result in focal or concentric left ventricular hypertrophy with myocardial disarray, fibrosis and extracellular matrix accumulation.

Hypertrophic cardiomyopathy is unique and presents with myriad of clinical symptoms and signs affecting infants to the very old $[1,4,5]$. Most of the affected individuals probably have an uneventful life. However, in some patients with HCM the disease results in profound clinical implications including 
sudden cardiac death (SCD), heart failure (HF), and atrial fibrillation with related complications. SCD due to ventricular tachyarrhythmias is the leading cause of death among relatively asymptomatic young adults with hypertrophic cardiomyopathy $[6,7]$. The only proven method for prevention of SCD is the implantation of Cardioverter-Defibrillator (ICD) [8]. In a study by Maron, et al. [8], 20\% of the study population with HCM received appropriate ICD intervention for life threatening arrhythmias during a mean follow-up of less than four years. These findings suggest that the risk factor strategy used in this study is a useful guide for identifying patients with HCM who are susceptible to sudden cardiac death but the $80 \%$ of the patients received 'inappropriate' ICD intervention.

Cardiovascular magnetic resonance imaging (CMR) provides optimal differentiation of normal and abnormal myocardium with use of late gadolinium enhancement (LGE) technique and special magnetic pulsed sequences $[9,10]$. The distribution of scar found on LGE-CMR in HCM patients had a strong correlation with fibrosis reported on histopathology [11]. Myocardial fibrosis detected by LGE-CMR in HCM patients was associated with increased frequency of ventricular tachyarrhythmias including NSVT on 24 hour Holter ECG [11,12]. In a recent CMR study by Kwon, et al. [13] demonstrated that HCM patients with VT had significantly higher scar percentage (\%), compared to those without VT ( $14 \%$ vs. $6 \%, p=0.03$ ). Myocardial scar visualized by CMR predicts major adverse events in patients with hypertrophic cardiomyopathy. The presence of scar indicated by CMR is good independent predictor of all-cause mortality, cardiac mortality and sudden cardiac death [14]. In the same study by Bruder, et al. [14] also demonstrated that absence of LGE in these patients had the highest negative predictive value.

The current risk stratification strategy to prevent SCD among patients with HCM involves identification of the following risk factors [15]. 1. A personal history of SCD event, VT/VF. 2. A family history of SCD events, including appropriate ICD therapy for ventricular arrhythmias. 3. Unexplained syncope. 4. Documented NSVT defined as 3 or more beats at greater than or equal to $120 \mathrm{bpm}$ on ambulatory Holter monitor. 5. Maximal LV wall thickness equal or greater than to $3 \mathrm{~cm}$. 6. Inappropriate blood pressure response to exercise. The criteria for an ICD implantation in HCM patients are still limited by clinical markers. In a recent study by Maron, et al. [8], appropriate ICD discharge was similar in patients in with 1,2 or 3 or more risk factors $(3.83,2.65$ and 4.82 per 100 person-years respectively). An important proportion of these device (ICD) interventions occurred in patients with single risk factor. Thus conceptually, a single risk factor may justify ICD consideration for a primary prevention in selected patients with HCM. There are still some questions that current clinical risk stratification cannot answer. One risk factor based ICD implantation strategy may exceed the number of patients expected to die suddenly. However, not all patients with 1 risk factor are at the same magnitude of risk and universal devise implantation is not recommended in these patients. The primary objective of this systematic review is to evaluate myocardial fibrosis detected by LGE-CMR as a risk factor for SCD in patients with hypertrophic cardiomyopathy.

\section{Methods}

We performed an electronic search of Medline, PubMed and CMR abstracts of original data published or presented between January 2001 and December 2011. The key search terms were HCM, left ventricular (LV) fibrosis, SCD and LGE. The inclusion criteria were referral for CMR exam with LGE for HCM and follow-up for incidence of reported VT/VF or SCD. The relevant studies were summarized and the following data were abstracted: 1. Socio-demographic information. 2. Study design. 3. The incidence of reported VT/VF or SCD. Categorical variables were evaluated between the groups by Chi-square test.

\section{Statistical methods}

The clinical and demographic patient characteristics from the included studies were summarized using simple descriptive statistics. Continuous variables were reported as mean + standard deviation; categorical variables as frequency or percent. Presence/absence of fibrosis was compared against VT/VF events and sudden cardiac death using the chisquare test. Odds ratios and $95 \%$ confidence intervals were calculated. Data with a $p<0.05$ was considered to indicate statistical significance. Data was analyzed using PASW Statistics, version 18 (IBM/SPSS Inc., Chicago).

\section{Results}

A total of 64 studies involving CMR studies in HCM patients were initially identified through our Medline and PubMed search. Of these, only four studies [14,16-18] approximately $6.3 \%$ were included in the study because of our very stringent inclusion and exclusion criteria involving SCD, VT/VF and ventricular fibrosis in HCM patients (Table 1). There were one thousand and sixty three patients $(n=1063)$ with a range of 202 to 424 patients in these studies. There were three prospective and one retrospective study included in this systematic analysis. The mean follow was 856 days with 2796 patient years of follow up. The age range in this study was 2 to 90 years with $63 \%$ of them were male gender.

\begin{tabular}{|l|l|c|c|c|c|c|c|}
\hline \multicolumn{6}{|l|}{$\begin{array}{l}\text { Table 1: Comparison of } 4 \text { CMR studies with late gadolinium enhancement in HCM } \\
\text { patients that reported SCDs and VT/VF. }\end{array}$} \\
\begin{tabular}{|} 
Study \\
year
\end{tabular} & Authors & $\begin{array}{c}\text { No. of } \\
\text { patients }\end{array}$ & $\begin{array}{c}\text { Follow } \\
\text { up days }\end{array}$ & Study type & $\begin{array}{c}\text { Patients } \\
\text { with LGE }\end{array}$ & $\begin{array}{c}\text { Patients } \\
\text { with VF/ } \\
\text { VT }\end{array}$ & $\begin{array}{c}\text { Patients } \\
\text { with SCD }\end{array}$ \\
\hline 2008 & $\begin{array}{c}\text { Maron, et al. } \\
\text { [18] }\end{array}$ & 202 & 681 & Prospective & $111(55 \%)$ & 0 & 7 \\
\hline 2010 & $\begin{array}{c}\text { Rubinshtein, } \\
\text { et al. [16] }\end{array}$ & 424 & 516 & Retrospective & $239(56 \%)$ & 0 & 8 \\
\hline 2010 & $\begin{array}{c}\text { O'Hanlon, } \\
\text { et al. [17] }\end{array}$ & 217 & 1132 & Prospective & $136(63 \%)$ & 9 & 4 \\
\hline 2010 & $\begin{array}{c}\text { Bruder, et } \\
\text { al. [14] }\end{array}$ & 243 & 1090 & Prospective & $148(67 \%)$ & 0 & 11 \\
\hline
\end{tabular}


Late gadolinium enhancement (LGE) was detected in $59.6 \%(621 / 1063)$ of patient population, which is consistent with other published studies in the literature. Sustained VT/ VF was detected in $13 / 621$ (2.09\%) of the fibrosis group and $0.23 \%(1 / 428)$ of the fibrosis negative group. The association between the presence of fibrosis and VT/VF were analyzed using the Pearson correlation coefficient in these four studies and showed significant correlation $\left.\left(\mathrm{x}^{2}\right)(1)=6.5, p=0.01\right)$ (Table 2). Similar results were seen when the SCDs vs. fibrosis were analyzed in these studies (Table 3). There were 25 (4\%) sudden cardiac deaths in fibrosis group and $5(1 \%)$ in the non-fibrosis group. Upon statistical analysis it showed that there was a significant correlation between fibrosis and SCDs among patients with HCM by combing all the four available studies (Pearson $\left.\mathrm{X}^{2}(1)=6.62, p=0.01\right)$.

\section{Discussion}

The terms meta-analysis and systematic review are often used interchangeably, particularly in North America. The term systematic review should refer to the entire process of collecting, reviewing and presenting all the available evidence incorporating appropriate statistical methods, while the term 'meta-analysis' will refer to the statistical technique in extracting and combining data to produce a summary result. A meta-analysis is a two-stage process. The first stage is the extraction of data from each individual study and the calculation of a result for that study. The second stage involves deciding whether it is appropriate to calculate pooled average result across studies and, if so calculating and presenting such a result typically in terms of a Forest plot. Based on statistical convention a systematic review was performed.

Hypertrophic cardiomyopathy is characterized by myocyte disarray and cardiac fibrosis resulting in asymmetrical or circumferential left ventricular hypertrophy. The most common clinical symptoms in HCM patients are dyspnea, angina, and presyncope or syncope. The most ominous and feared complication in these patients especially in young adults is sudden cardiac death in that no uniform metric reliably predicts risk, clinical metrics are substituted to represent risk.

Table 2: Fibrosis vs. SCD, The association between the presence of fibrosis and SCD was statistically significant Pearson $x^{2}(1)=6.62, p=0.01$. Power of the performed test with $p=0.05$ is 0.67 Odds ratio: $3.34 ; 95 \% \mathrm{Cl} 1.26$ to 8.81 .

\begin{tabular}{|c|c|c|c|}
\hline & \multicolumn{3}{|c|}{ SCD } \\
\hline Fibrosis & Yes & No & Total \\
\hline Yes & 25 & 610 & 635 \\
\hline No & 5 & 424 & 429 \\
\hline Totals & 30 & 1034 & 1064 \\
\hline
\end{tabular}

Table 3: Fibrosis vs. VT/VF Events, The association between the presence of fibrosis and SCD was statistically significant Pearson $x^{2}(1)=6.5, p=0.01$. Power of the performed test with alpha $=0.05$ is 0.62 . Odds ratio: $8.96 ; 95 \% \mathrm{Cl} 1.17$ to 68.75 .

\begin{tabular}{|c|c|c|c|}
\hline & \multicolumn{3}{|c|}{ VT/VF Events } \\
\hline Fibrosis & Yes & No & Total \\
\hline Yes & 13 & 621 & 634 \\
\hline No & 1 & 428 & 429 \\
\hline Totals & 14 & 1049 & 1063 \\
\hline
\end{tabular}

The risk factors for SCD in these patients are personal history of cardiac arrest, family history of SCD, episodes of recurrent unexplained syncope, an abnormal blood pressure response with exercise, non-sustained ventricular tachycardia, and a maximum LV wall thickness greater than $3 \mathrm{~cm}$ [19]. The most common cause of SCD is ventricular tachycardia/fibrillation. Cardiac fibrosis has been demonstrated as the substrate for induction and maintenance of ventricular tachyarrhythmia [12]. Currently, the best means of preventing SCD and prolonging life in HCM patients is implanting an ICD [8].

The clinical diagnosis of HCM is conventionally performed with cardiac imaging, most commonly with 2-D echocardiography. However, CMR has superior spatial resolution (spatial density and optical resolution) and can delineate the entire LV myocardium and therefore can more accurately characterize the presence and distribution of LV hypertrophy especially those with focal hypertrophy as seen HCM patients. Contrast enhanced CMR with LGE sequences can accurately identify areas of myocardial fibrosis, which has been implicated in heart failure as well as substrate for ventricular arrhythmias $[11,12,20]$. The incidence of non-sustained ventricular tachycardia is high in HCM patients with myocardial fibrosis and is an independent predictor of sudden cardiac death in these patients $[12,17,21]$. These studies would suggest that LGE might represent a substrate for malignant ventricular arrhythmias and subsequent SCD.

The current risk stratification strategy using the five major risk factors to prevent SCD in HCM remains incomplete in spite of significant advances in the molecular genetics and newer imaging techniques. Late gadolinium enhancement (LGE) representing myocardial fibrosis in these disease patients could represent an ideal risk marker in predicting sudden cardiac death in HCM patient and thereby improving risk stratification by itself or in combination with current risk factors. In view of this question, we searched the currently available literature for hypertrophic cardiomyopathy patients having incorporated a clinical assessment in HCM patients via CMR-LGE with long term follow-up for SCD as related to myocardial fibrosis and found 3 prospective and one retrospective study (Table 1) to answer our question. The summery of these studies are discussed in the following section.

Maron, et al. [18], in their 2007 study reported that LGE was not a sufficient risk factor for adverse prognosis. They prospectively followed 202 patents over period of $681 \pm 249$ days and found that LGE was positive in $55 \%$ of their cohorts. Adverse cardiovascular events occurred in $11 / 220$ patients. Of these patients with adverse cardiovascular events, 7 were positive for LGE (63.6\%). Two patients out of seven with fibrosis (28.6\%) suffered SCD and another two patients (28.6\%) had ICD discharge to abort SCD. In LGE negative cohort 4 patients died and 3 of them (75\%) suffered sudden cardiac death. Over the follow-up period, the annualized 
adverse CV event rate in patients with LGE exceeded that in patients without LGE but did not reach statistical significance (5.5\% vs. 3.3\%, HR 1.45, 95\% CI 0.43 to 4.97). Extent of LGE did not differ between patients with and without adverse CV events $(9 \pm 11 \%$ vs. $11 \pm 15 \%, p=0.97)$.

O'Hanlon, et al (prospectively followed 217 patients with HCM over a period of $3.1 \pm 1.7$ years and found myocardial fibrosis in $63 \%(n-136)$ of patients. The combined primary end points (CV death, unplanned hospital stay, sustained VT/ $\mathrm{VF}$, appropriate ICD discharge) were reached in $25 \%$ of the fibrosis group and $7.4 \%$ of fibrosis negative group. During follow-up there were 9 cardiovascular deaths, 8 of which (89\%) occurred in the fibrosis group. There were 2 SCD with one SCD in each group. Overall, 40/217 (18.4\%) patients reached the primary end point: $34 / 136(25 \%)$ in the fibrosis group, and 6/81 (7.4\%) in the non-fibrosis group (HR: 3.4, $95 \% \mathrm{CI} 1.4$ to $8.1, p=0.006)$. In the fibrosis group, the overall risk of the primary endpoint increased with the increased percentage of fibrosis (HR 1.18/5\% fibrosis increase, 95\% CI: 105 to $1.33, p=0.008$ ). For every $5 \%$ increase in fibrosis, the risk of reaching the primary endpoint increased by $15 \%$. In vivo fibrosis detection by LGE is an independent predictor of an adverse outcome in this disease.

Bruder, et al. [14], in their recently published article on "the role of myocardial scar visualized by CMR in patients with hypertrophic cardiomyopathy" demonstrated that LGE independently carries an adverse risk of high cardiac mortality. They prospectively followed 220 HCM patients (mean age of 58 years) over a period of 1090 days after the initial CMR exam. LGE was present in 148 (67.3\%) of 220 patients. In general, there was no difference between scar and no scar patients except higher burden of hypertrophy as well as history of arrhythmias in the scar population. During the follow-up, 20/220 (9\%) patients died, of which 16 were due to cardiac events. There were 8 SCDs and 2 aborted SCDs in the LGE group and one SCD in the LGE negative cohort. This data indicates that the presence of scar as visualized by LGE- CMR yields an odds ratio of 5.47 for all-cause mortality and 8.01 for cardiac mortality. This might be superior to current risk factor strategy because of presence of 2 risk factors yields an odds ratio of 3.86 for all cause and 2.20 for cardiac mortality. Multivariate analysis also revealed the presence of cardiac fibrosis revealed by LGE is a good independent predictor of death in HCM patients.

Rubinshtein, et al. [16], in their retrospective study involving 424 HCM patients reported that 239 patients (56\%) were positive for LGE. The mean age of the study population was $55 \pm 16$ years with age range from 2 to 90 years and 251 of them (59.2\%) were male. During the follow-up period, 11 patients died, and all were positive for LGE. SCD occurred in 4 patients, and additional 4 patients received appropriate ICD discharges. Estimated event-free survival at 6 years was $100 \%$ in patients without LGE compared with $96 \%$ in
LGE positive group (log rank, $p=0.01)$. Univariate analysis revealed that LGE was significantly associated with outcome events (0.002), however, a hazard ratio for LGE was not calculated because all events were in the LGE positive group. LGE was strongly associated with arrhythmia and remained a significant associate of subsequent SCD and/or ICD discharge after controlling for other variables.

Despite several single center CMR studies that consistently predicted VT/VF based on LGE sequences, the prediction of SCD is still remains uncoupled in HCM patients. Cardiac MRI with late gadolinium enhancement to visualize fibrosis/scar is relatively a new technique, and for the first time, in this systematic review, sudden cardiac death could be predicted by its presence or absence. This observation now demands a multi-center randomized clinical trial for confirmation but supports the consideration of primary indication or as an adjudicator in borderline risk patients for an AICD implantation in hypertrophic cardiomyopathy patients who are positive for LGE.

\section{Conclusion}

Several single center LGE-CMR studies had consistently predicted VT/VF in HCM patients but prediction of SCD in these remained paradoxically unlinked. Although there were limited number of studies meeting our criteria that limited our ability to conduct a comprehensive meta-analysis, we have been able to demonstrate for the first time that LGE-defined fibrosis/scar is a predictor of SCD in patients with HCM.

\section{Study Limitations}

The lack of studies meeting our criteria limited our ability to perform a comprehensive meta-analysis. Small number of patients in these studies with sudden cardiac death and fibrosis also limits the statistical power of the study. HCM patients who have already received AICD were excluded from these studies and thus precluding these high-risk patients from participation and influencing the study results. Finally this is not a randomized trial, which is considered as the highest level of scientific evidence. However, systematic reviews of RCTs also provide better quality of evidence that is available at this point in time.

\section{Disclosures}

All the authors have none to disclose except off label use of gadolinium.

\section{References}

1. Maron BJ, Gardin JM, Flack JM, Gidding SS, Kurosaki TT, et al. Prevalence of hypertrophic cardiomyopathy in a general population of young adults. Circulation. 1995; 92: 785-789.

PubMed: https://www.ncbi.nlm.nih.gov/pubmed/7641357

2. Marian AJ, Roberts R. Recent advances in the molecular genetics of hypertrophic cardiomyopathy. Circulation. 1995; 92: 1336-1347. PubMed: https://www.ncbi.nlm.nih.gov/pubmed/7648684 
3. Alcalai R, Seidman JG, Seidman CE. Genetic basis of hypertrophic cardiomyopathy: from bench to the clinics. J Cardiovasc Electrophysiol. 2008;19:104-10.

PubMed: https://www.ncbi.nlm.nih.gov/pubmed/17916152

4. Wigle ED, Rakowski H, Kimball BP, Williams WG. Hypertrophic cardiomyopathy: clinical spectrum and treatment. Circulation. 1995; 92: 1680-1692.

PubMed: https://www.ncbi.nlm.nih.gov/pubmed/7671349

5. Maron BJ. Hypertrophic cardiomyopathy: A systematic review. JAMA 2002; 287: 1308-1320.

PubMed: https://www.ncbi.nlm.nih.gov/pubmed/11886323

6. Elliott PM, Poloniecki J, Dickie S, Sharma S, Monserrat L, et al. Sudden death in hypertrophic cardiomyopathy: identification of high risk patients. J Am Coll Cardiol. 2000 ;36: 2212-2218.

PubMed: https://www.ncbi.nlm.nih.gov/pubmed/11127463

7. Maron BJ, Olivotto I, Spirito P, Casey SA, Bellone P, et al. Epidemiology of hypertrophic cardiomyopathy-related death: revisited in a large nonreferral-based patient population. Circulation. 2000; 102: 858-864. PubMed: https://www.ncbi.nlm.nih.gov/pubmed/10952953

8. Maron BJ, Spirito P, Shen WK, Haas TS, Formisano F, et al. Implantable Cardioverter-Defibrillators and prevention of sudden cardiac death in hypertrophic cardiomyopathy. JAMA. 2007; 298: 405-412.

PubMed: https://www.ncbi.nlm.nih.gov/pubmed/17652294

9. Biederman RWW, Doyle M, Yamrozik J. Cardiovascular MRI tutorials: Lectures and Learning. Lippincott Williams et al. \& Wilkins 2007.

10. Amin NN, Grant SB, Yamrozik JA, Williams RB, Thompson DV, et al. The Concordance Rates between LV Hypertrophy and RV Hypertrophy in Patients with Hypertrophic Cardiomyopathy as Diagnosed by Cardiovascular MRI with Fibrosis Imaging. World $\mathrm{J}$ of Cardiovasc Dis. 2015; 5: 171-180.

11. Kwon DH, Smedira NG, Rodriguez ER, Tan C, Setser R, et al. Cardiac magnetic resonance detection of myocardial scarring in hypertrophic cardiomyopathy: correlation with histopathology and prevalence of ventricular tachycardia. J Am Coll Cardiol. 2009; 54: 242-249.

PubMed: https://www.ncbi.nlm.nih.gov/pubmed/19589437

12. Adabag AS, Maron BJ, Appelbaum E, Harrigan CJ, Buros JL, et al. Occurrence and frequency of arrhythmias in hypertrophic cardiomyopathy in relation to delayed enhancement on cardiovascular magnetic resonance. J Am Coll Cardiol. 2008; 51: 1369-1374.

PubMed: https://www.ncbi.nlm.nih.gov/pubmed/18387438
13. Kwon DH, Setser RM, Popović ZB, Thamilarasan M, Sola S, et al. Association of myocardial fibrosis, electrocardiography and ventricular tachyarrhythmia in hypertrophic cardiomyopathy: a delayed contrast enhanced MRI study. Int J Cardiovasc Imaging 2008;24:617-625. PubMed: https://www.ncbi.nlm.nih.gov/pubmed/18204915

14. Bruder O, Wagner A, Jensen CJ, Schneider S, Ong P, et al. Myocardial scar visualized by cardiovascular magnetic resonance imaging predicts major adverse events in patients with hypertrophic cardiomyopathy. J Am Coll Cardiol. 2010; 56: 875-887.

PubMed: https://www.ncbi.nlm.nih.gov/pubmed/20667520

15. Gersh BJ, Maron BJ, Bonow RO, Dearani JA, Fifer MA, et al. 2011 ACCF/AHA Guidelines for the diagnosis and treatment of hypertrophic cardiomyopathy: a report for the American College of Cardiology Foundation/American Heart Association Task Force on Practice Guidelines. J Am Coll Cardiol. 2011; 58: e212-260.

PubMed: https://www.ncbi.nlm.nih.gov/pubmed/22068435

16. Rubinshtein R, Glockner JF, Ommen SR, Araoz PA, Ackerman MJ, et al. Characteristics and clinical significance of late gadolinium enhancement by contrast magnetic resonance imaging in patients with hypertrophic cardiomyopathy. Circ Heart Fail. 2010; 3: 51-58. PubMed: https://www.ncbi.nlm.nih.gov/pubmed/19850699

17. O'Hanlon R, Grasso A, Roughton M, Moon JC, Clark S, et al. Prognostic significance of myocardial fibrosis in hypertrophic cardiomyopathy. $J$ Am Coll Cardiol. 2010; 56: 867-874.

PubMed: https://www.ncbi.nlm.nih.gov/pubmed/20688032

18. Maron MS, Appelbaum E, Harrigan CJ, Buros J, Gibson CM, et al. Clinical profile and significance of delayed enhancement in hypertrophic cardiomyopathy. Circ Heart Fail. 2008; 1: 184-191.

PubMed: https://www.ncbi.nlm.nih.gov/pubmed/19808288

19. Elliot P, McKenna WJ. Hypertrophic cardiomyopathy. Lancet 2004; 363: 1881-1991.

PubMed: https://www.ncbi.nlm.nih.gov/pubmed/15183628

20. Olivotto I, Maron BJ, Appelbaum E, Harrigan CJ, Salton C, et al. Spectrum and clinical significance of systolic function and myocardial fibrosis assessed by cardiovascular magnetic resonance in hypertrophic cardiomyopathy. Am J Cardiol 2010;106:261-267.

PubMed: https://www.ncbi.nlm.nih.gov/pubmed/20599013

21. Monserrat L, Elliott PM, Gimeno JR, Sharma S, Penas-Lado M, et al Non-sustained ventricular tachycardia in hypertrophic cardiomyopathy: An independent marker of sudden death risk in young patients. J Am Coll Cardiol 2003; 42: 873-879.

PubMed: https://www.ncbi.nlm.nih.gov/pubmed/12957435 